\title{
Polynomial Methods in Noisy Network Localization
}

\author{
Iman Shames, Pouyan Taghipour Bibalan, Barış Fidan, and Brian D. O. Anderson \\ The Australian National University and National ICT Australia, Canberra, Australia \\ Email: \{Iman.Shames,u4422921,Baris.Fidan,Brian.Anderson\} @anu.edu.au
}

\begin{abstract}
In this paper we introduce a polynomial method for addressing sensor network localization problems when the inter-sensor measurements are noisy. We compare the results obtained applying this method with the results obtained by other localization methods in the literature. Later in the paper we propose tools from algebraic geometry to aid us solve the problem in a more computationally appealing way.
\end{abstract}

\section{INTRODUCTION}

In many applications, knowing the positions of the sensors in a sensor network accurately is essential. For example, if the sensor network is deployed to monitor a region of interest, it is important to be able to relate the sensory data to a location in the environment, or in communication networks in order to select a route for the data communication to minimize the used energy by the nodes it is necessary to know the position of the nodes. A simple solution to this problem is using the global positioning system (GPS) receivers at each node, however, this approach faces three major shortcomings; first, for a large network it will be financially infeasible to install a GPS receivers at each node. Second, GPS receivers draw on the energy source of the nodes and require use of energy cells with higher capacity or replacing the energy cells more frequently. Third, GPS sensors are vulnerable to several categories of signal disruptions; intentional (e.g. jamming and spoofing) or unintentional (eg. radio frequency interference from radio, $\mathrm{TV}$, cell phones and other communication transmitters) [1]. Another related problem is localizing a target of interest using range measurements obtained from a set of sensors, see [2] and the references therein for more information.

The examples above demonstrate the need for using methods to calculate the position of the nodes based on sensory data, noting that there are various other issues, like these examples, in different settings as well. A widely used approach to the problem of network localization is to use distance measurements from the node whose position is of interest to other nodes or landmarks with known positions, anchors (beacons), and infere the position of the node based on the obtained information. For the sake of clarity we describe a scenario in what follows.

Consider the nodes are scattered in an $N$-dimensional space, $N \in\{2,3\}$. Consider $n>N$ anchor nodes, labeled $1,2, \ldots, n$. Assume each (anchor) node $i \in\{1, \cdots, n\}$ are at a generic position $p_{i} \in \mathbb{R}^{N} ; p_{i}=\left[x_{i}, y_{i}\right]^{\top}$ for $N=2$ and $p_{i}=\left[x_{i}, y_{i}, z_{i}\right]^{\top}$ for $N=3$. Here by having generic positions, we mean that for $N=2$ no triple of anchor nodes are collinear, and for $N=3$ no quadruple of anchor nodes are coplanar. Consider a non-anchor sensor node, node 0 ,

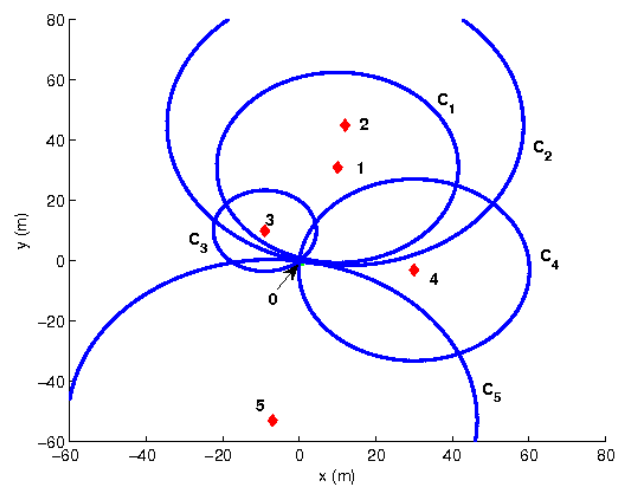

Fig. 1. Distance based localization problem.

placed at unknown position $p_{0}=p^{*}=\left[x^{*}, y^{*}\right]^{\top}$, which, and can measure its distance from anchor nodes $1,2, \ldots, n$, i.e. it measures $d_{i}^{*}=\left\|p_{i}-p^{*}\right\|$, for each $i \in\{1, \cdots, n\}$. Note that, in this paper we denote the actual distance $\left\|p_{i}-p\right\|$ by $d_{i}^{*}$ and the corresponding measurement by $d_{i}$. The problem of interest is finding an accurate estimate $p$ for $p^{*}$ using the measurements $d_{i}$.

If the measurements are precise $\left(d_{i}=d_{i}^{*}\right)$ the solution is trivial: $p=p^{*}$ is the unique point of intersection of the $n$ circles, $C_{i}$ :

$$
C_{i}:\left\|p_{i}-p\right\|^{2}-d_{i}^{2}=0, \quad i \in\{1, \cdots, n\} .
$$

The setting is illustrated in Fig. 1. However, unfortunately, it is never the case that the measurements agree with the actual distances. Furthermore, we know that when the measurements are noisy, the circles may not have a common point of intersection, so one needs another method to solve the problem.

There are some remedies in the literature to deal with this problem. In [3] a linear algorithm to tackle the problem is proposed. Later in [4] a non-linear method using Cayley-Menger determinant is proposed and its performance is compared with that of [3], which is shown to have better results. Another paper that has considered application of Cayley Menger determinant to solve localization problems is [5], where an in-depth error analysis in case of having three anchors is presented. The closest result in the literature to that of this paper is the one presented in [2], where the authors considered least squares (LS) approaches for locating a radiating source from range measurements (R-LS) and squared range measurements (SRLS).

In the literature, the relationship between the distance 
measurements $d_{i}$ and the actual distances $d_{i}^{*}$ is modeled in various ways, e.g. $d_{i}=d_{i}^{*}+\varepsilon_{i}$ [6], $d_{i}^{2}=d_{i}^{* 2}+\varepsilon_{i}^{2}$ [4], or $\ln d_{i}=\ln d_{i}^{*}+\varepsilon_{i}$ [7], where $\varepsilon_{i}$ denotes a measurement error term. Some of these models are simplistic and rather fictitious, while the others are derived from the physics of the mechanism used in distance measurement. Modelling of the error terms $\varepsilon_{i}$ is a part of the general distance measurement modeling as well, and can be done in various ways [4], [6], [7]; nevertheless, they are in general modeled as random variables with certain distributions such as Gaussian or log-normal [8].

The paper outline is as follows. First, we provide some preliminary information on algebraic geometry in Section II. In Section III we formally define the distance measurement based localization problem of interest using distance measurements in two-dimensional and three-dimensional spaces. In Section IV we propose a method to solve localization problem using range difference measurements. In Section $\mathrm{V}$, an alternative method using sum of squares for solving the problem of interest is presented. In Section VI, some numerical examples are presented. The method presented here is compared with the other methods in the literature in Section VII. In Section VIII, we consider the localization problem in a non-generic case where the anchors are colinear. In the end concluding remarks and future work is presented in Section IX.

\section{Preliminaries}

In this section we introduce some basic notions from algebraic geometry that we use later in the paper. We use $\mathbb{R}\left[x_{1}, \cdots, x_{n}\right]$ to denote the ring of all polynomials in $n$ indeterminants (variables), $x_{1}, x_{2}, \cdots, x_{n}$. with real coefficients. The set $\mathcal{I} \subset \mathbb{R}\left[x_{1}, \cdots, x_{n}\right]$ is an ideal if $q h \in \mathcal{I}$ for any $q \in \mathcal{I}$ and $h \in \mathbb{R}\left[x_{1}, \cdots, x_{n}\right]$. We write $\left\langle g_{1}, \cdots, g_{r}\right\rangle$ to represent the set of all polynomials that are polynomial linear combinations of the $g_{i}$.

Definition 2.1: Let $f_{1}, \ldots, f_{m}$ be polynomials in $\mathbb{R}\left[x_{1}, \cdots, x_{n}\right]$. Let the set $\mathcal{V}$ be

$$
\begin{aligned}
\mathcal{V}\left(f_{1}, \cdots, f_{m}\right)= & \left\{\left(a_{1}, \cdots, a_{n}\right) \in \mathbb{C}: f_{i}\left(a_{1}, \cdots, a_{n}\right)=0,\right. \\
& \forall 1 \leq i \leq m\} .
\end{aligned}
$$

We call $\mathcal{V}\left(f_{1}, \cdots, f_{m}\right)$ the variety defined by $f_{1}, \cdots, f_{m}$. Then, the set of polynomials that vanish in a given variety, i.e.,

$$
\begin{aligned}
\mathcal{I}(\mathcal{V})= & \left\{f \in \mathbb{R}\left[x_{1}, \cdots, x_{n}\right]: f\left(a_{1}, \cdots, a_{n}\right)=0\right. \\
& \left.\forall\left(a_{1}, \cdots, a_{n}\right) \in \mathcal{V}\right\},
\end{aligned}
$$

is an ideal, called the ideal of $\mathcal{V}$. Furthermore, the subset of all real points in $\mathcal{V}\left(f_{1}, \cdots, f_{m}\right)$ is the real variety defined by $f_{1}, \cdots, f_{m}$, i.e.

$$
\begin{aligned}
\mathcal{V}_{\mathbb{R}}\left(f_{1}, \cdots, f_{m}\right)= & \left\{\left(a_{1}, \cdots, a_{n}\right) \in \mathbb{R}: f_{i}\left(a_{1}, \cdots, a_{n}\right)=0,\right. \\
& \forall 1 \leq i \leq m\} .
\end{aligned}
$$

A polynomial function $F(x)$ of degree $2 d$, over the polynomial $\operatorname{ring} \mathbb{R}\left[x_{1}, \cdots, x_{n}\right]$ is sum of squares (SOS) if one can write,

$$
F(x)=\sum_{i=1}^{q} Q_{i}^{2}(x)
$$

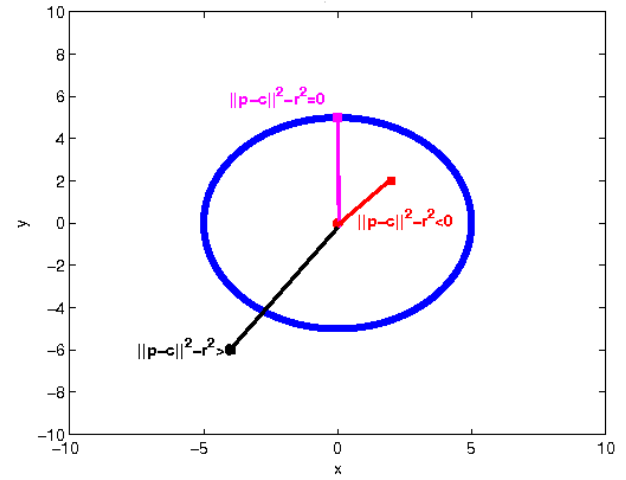

Fig. 2. Powers of different points with respect to circle $x^{2}+y^{2}=r^{2}$.

where $q \in \mathbb{Z}^{+}$and $Q_{i}(x)$ are the polynomials over $\mathbb{R}\left[x_{1}, \cdots, x_{n}\right]$.

\section{Distance Measurement Based Localization}

First we formally define the problem of interest.

Problem 3.1: Consider $n$ anchor nodes in $\mathbb{R}^{N}(N \in\{2,3\})$ at the known positions $p_{i}, i \in\{1, \cdots, n\}$, and a sensor node 0 at the unknown position $p^{*}$. Let the noisy measurement $d_{i}$ of the distance of node 0 to each node $i$ for $i \in\{1, \ldots n\}$ be available (to node 0 ). The task (of node 0 ) is to produce the estimate $p$ of $p^{*}$ using the noisy distance measurements $d_{1}, \cdots, d_{n}$.

\section{A. Localization in $\mathbb{R}^{2}$}

First we consider the case $N=2$. Let us revisit the problem of interest from a geometric point of view. In noiseless case the intersection of the circles is the point that satisfies all the circle equations (1). Before going further, we define power of a point, say $p$, with respect to a circle, $C$ with center at $c$ and radius $r$ as

$$
P(p, C)=\|p-c\|^{2}-r^{2},
$$

Note that $P(p, C)>0$ if $p$ is outside the circle, $P(p, C)<0$ if $p$ in inside the circle, and $P(p, C)=0$ if $p$ is on the circle. These different cases are depicted in Fig. 2. So, for the point of intersection, $p$ we have,

$$
P\left(p, C_{i}\right)=0, \quad i \in\{1, \cdots, n\}
$$

Or equivalently,

$$
\begin{aligned}
J^{*}(p) & =\sum_{i=1}^{n} P^{2}\left(p, C_{i}^{*}\right) \\
& =\sum_{i=1}^{n}\left(\left\|p-p_{i}\right\|^{2}-d_{i}^{* 2}\right)^{2}=0
\end{aligned}
$$

where $C_{i}^{*}$ denotes $C_{i}$ for $d_{i}=d_{i}^{*}$, and $C_{i}$ and $d_{i}^{*}$ are as defined in Section I.

Remark 3.1: Function $J(p)$ is positive semidefinite, in other words $J(p) \geq 0$ for all $p \in \mathbb{R}^{2}$.

Remark 3.2: Function $J(p)$ has exactly one root for generic positions of $p_{i}$ ( $p_{i}$ non-collinear). Moreover, this root is the 
global minimizer of the function $J$. Hence, instead of finding the root of $J(p)$, one can find the global minimizer of $J(p)$. Now consider the noisy case where $d_{i} \neq d_{i}^{*}$. Having the same geometric view as before, we are interested in finding the point $p$ which minimizes the following modified version of (5):

$$
\begin{aligned}
J(p) & =\sum_{i=1}^{n} P^{2}\left(p, C_{i}\right) \\
& =\sum_{i=1}^{n}\left(\left\|p-p_{i}\right\|^{2}-d_{i}^{2}\right)^{2} .
\end{aligned}
$$

In general $J(p)$ is not convex (or concave), however, using elementary calculus one can find the critical points of $J(p)$ by finding $[x, y]^{\top}$ which satisfies,

$$
\frac{\partial J}{\partial x}=\frac{\partial J}{\partial y}=0
$$

The system (7) has a maximum of $m \leq 9$ pairs of real solutions [9]. Denote these solutions by $\bar{p}_{k}=\left[\bar{x}_{k}, \bar{y}_{k}\right]^{\top}$, $k \in\{1, \cdots, m\}$. Furthermore, define the set $\mathcal{V}_{\mathbb{R}}\left(\frac{\partial J}{\partial x}, \frac{\partial J}{\partial y}\right)=$ $\left\{\bar{p}_{k}\right\}_{k=1}^{m}$. The desired minimizer point can be calculated as the solution of

$$
p=\underset{p \in \mathcal{V}_{\mathbb{R}}\left(\frac{\partial J}{\partial x}, \frac{\partial J}{\partial y}\right)}{\operatorname{argmin}}\left(\sum_{i=1}^{n} P^{2}\left(p, C_{i}\right)\right) .
$$

\section{B. Localization in $\mathbb{R}^{3}$}

In this subsection we extend the method in Section III.A to the cases where the sensors are deployed in a threedimensional space. The cost function is defined as

$$
J(p)=\sum_{i=1}^{n}\left(\left\|p-p_{i}\right\|^{2}-d_{i}^{2}\right)^{2} .
$$

Here the problem is to find the real variety of the ideal $\mathcal{I}_{3}=$ $\left\langle\frac{\partial J}{\partial x}, \frac{\partial J}{\partial y}, \frac{\partial J}{\partial z}\right\rangle$, or equivalently $\mathcal{V}_{\mathbb{R}}\left(\frac{\partial J}{\partial x}, \frac{\partial J}{\partial y}, \frac{\partial J}{\partial z}\right)$; and the point of interest is the solution of

$$
p=\underset{p \in \mathcal{V}_{\mathbb{R}}\left(\frac{\partial J}{\partial x}, \frac{\partial J}{\partial y}, \frac{\partial J}{\partial z}\right)}{\operatorname{argmin}}\left(\sum_{i=1}^{n}\left(\left\|p-p_{i}\right\|^{2}-d_{i}^{2}\right)^{2}\right) .
$$

\section{Localization Via RANGe DifFERENCE} Measurements in the Presence of Noise

We use the problem formulation presented in [2]. Suppose $n$ anchors are scattered as before in $\mathbb{R}^{N}(N \in\{2,3\})$. In addition, another anchor, $n+1$ is at the origin and the range difference measurements between sensor $n+1$ and sensor $i$ are (without explicitly knowing $d_{i}$ or $\|p\|$ ), $\delta_{i}$ is the measurement of $\delta_{i}^{*}=d_{i}^{*}-\left\|p^{*}\right\|, i=1, \cdots, n$, which can be written as

$$
\begin{aligned}
\delta_{i}^{* 2} & =d_{i}^{* 2}+\left\|p^{*}\right\|^{2}-2\left\|p^{*}\right\| d_{i}^{*} \\
\delta_{i}^{* 2} & =\left\|p_{i}\right\|^{2}+2\left\|p^{*}\right\|^{2}-2 p_{i}^{\top} p-2\left\|p^{*}\right\| d_{i}^{*} \\
\delta_{i}^{* 2}-\left\|p_{i}\right\|^{2} & =2\left\|p^{*}\right\|\left(\left\|p^{*}\right\|-d_{i}^{*}\right)-2 p_{i}^{\top} p \\
\delta_{i}^{* 2}-\left\|p_{i}\right\|^{2} & =-2 \delta_{i}^{*}\left\|p^{*}\right\|-2 p_{i}^{\top} p .
\end{aligned}
$$

However, when the range difference measurements are noisy these equations do not hold. One can define the following problem.

Problem 4.1: Consider $n$ anchor nodes in $\mathbb{R}^{N}(N \in\{2,3\})$ at the known positions $p_{i}, i \in\{1, \cdots, n\}$, another node $n+1$ is at the origin, and sensor node 0 at the unknown position $p^{*}$. Let $\delta_{i}$, the noisy measurement of $\delta_{i}^{*}$, be available (to node 0 ). The task (of node 0 ) is to produce the estimate $p$ of $p^{*}$ using the noisy range difference measurements $\delta_{1}, \cdots, \delta_{n}$.

To solve Problem 4.1 one is interested in solving the following minimization problem:

$$
\operatorname{minimize} \sum_{i=1}^{n}\left(\delta_{i}^{2}-\left\|p_{i}\right\|^{2}+2 \delta_{i}\|p\|+2 p_{i}^{\top} p\right)^{2} .
$$

Denoting $\|p\|$ by $D$, and considering that $D^{2}-x^{2}-y^{2}=0$ the problem will be transformed to

$$
\begin{array}{ll}
\text { minimize } & \sum_{i=1}^{n}\left(\delta_{i}^{2}-\left\|p_{i}\right\|^{2}+2 \delta_{i} D+2 p_{i}^{\top}\left[\begin{array}{l}
x \\
y
\end{array}\right]\right)^{2} \\
\text { subject to } & D^{2}-x^{2}-y^{2}=0
\end{array}
$$

Using Lagrange multiplier method we solve

$$
\begin{aligned}
\operatorname{minimize} & \sum_{i=1}^{n}\left(\delta_{i}^{2}-\left\|p_{i}\right\|^{2}+2 \delta_{i} D+2 p_{i}^{\top}\left[\begin{array}{l}
x \\
y
\end{array}\right]\right)^{2} \\
& -\lambda\left(D^{2}-x^{2}-y^{2}\right) .
\end{aligned}
$$

Note that if the solution of (14) corresponds to a negative $D$, we discard this result and choose another minimizer of $\sum_{i=1}^{n}\left(\delta_{i}^{2}-\left\|p_{i}\right\|^{2}+2 \delta_{i} D+2 p_{i}^{\top}\left[\begin{array}{l}x \\ y\end{array}\right]\right)^{2}$ with positive $D$.

\section{Sum of Square Methods and SDP}

As stated earlier to obtain the solution to problems like Problem 3.1 we need to solve a system of equations which consists of $N$ unknowns and $N$ equations of degree 3. The usual methods to solve such a system of nonlinear equations are based on Newton method, and there is not any guarantee that the solutions of the system are going to be found completely. However, there are other methods available to solve the problem of finding the global minimum of a polynomial function and in particular problems of the form (8). In this section we describe one of the methods that achieves the goal via sum of squares relaxation and semidefinite programming (SDP), see [10] and references therein.

Consider the two-dimensional case, where node 0 measures its distance, $d_{i}$, from nodes $i \in\{1, \cdots, n\}$, i.e. the case in Section III.A. One can easily show that $J$ is a sum of squares in the form of:

$$
J(p)=\sum_{i=1}^{n}\left(\left(x-x_{i}\right)^{2}+\left(y-y_{i}\right)^{2}-d_{i}^{2}\right)^{2}
$$

Call the global minimum of $J, J^{*}=\gamma$, and can be calculated solving the following optimization problem.

$$
\gamma^{*}=\max \gamma, \quad \text { subject to } J(p)-\gamma \geq 0 .
$$


One can relax (16) and write it as,

$$
\gamma^{*}=\max \gamma, \quad \text { subject to } J(p)-\gamma \text { is } \operatorname{SOS} \text {. }
$$

Remark 5.1: The relaxed problem is often computationally much easier to solve, and may yield the same solution. However, it is obvious that in general (16) and (17) are not identical, since there are positive polynomials that are not in the SOS form. For more information see [11].

We know that any polynomial $F(x)$ of degree $2 d$, with $x$ to be an $n$-tuple of variables, that is SOS can be written as $F(x)=Z^{\top} Q Z$, where $Z$ is a vector of all monomials of degree up to $d$ obtained from the variables in $x$ with the first entry equal to one, and $Q$ is a positive semi-definite matrix obtained by solving a set of linear matrix inequalities (LMI) [11]. So one can reformulate (17) as,

$$
\gamma^{*}=\max \gamma, \quad \text { subject to } \quad Q-\hat{E} \gamma \geq 0,
$$

where $\hat{E}$ is a matrix with $\hat{E}_{11}=1$ and the rest of the entries are zero. The problem stated in (18) is an SDP problem and can be solved by SDP techniques [11]. By solving the dual problem of (18), one can obtain the minimizer of $J$ as well, using the procedure in [12].

Remark 5.2: For polynomials with 2 variables and degree of 4 (16) and (17) are equivalent [11].

In the next section we show the application of SOS based methods to accomplish localization in a more complicated scenarios.

\section{NUMERICAL EXAMPLES}

In this section we show the applicability of our methods via numerical examples. In Examples 6.1-6.4 we consider the localization when range measurements are available, and in Example 6.5 we consider the case where range difference measurements are available. In Example 6.6 we provide an example where SOS relaxation is used to solve the problem.

Example 6.1: We use the same example as in [4]: Consider three anchor nodes at $p_{1}=[0,0]^{\top}, p_{2}=[43,7]^{\top}$ and $p_{3}=$ $[47,0]^{\top}$, and a sensor node zero using distance measurements from these anchors to localize itself.. The actual distances that are not available are $d_{1}^{*}=34.392, d_{2}^{*}=44.1106$, and $d_{3}^{*}=$ 41.2608 , while the noisy distance measurements by sensor 0 are $d_{1}=35, d_{2}=42$, and $d_{3}=43$. Hence,

$$
\begin{aligned}
J(p) & =\left(x^{2}+y^{2}-1225\right)^{2}+\left((x-43)^{2}+(y-7)^{2}-1764\right)^{2} \\
& +\left((x-47)^{2}+y^{2}-1849\right)^{2},
\end{aligned}
$$

and we solve,

$$
\begin{aligned}
\frac{\partial J}{\partial x} & =4\left(x^{2}+y^{2}-1225\right) x \\
& +2\left((x-43)^{2}+(y-7)^{2}-1764\right)(2 x-86) \\
& +2\left((x-47)^{2}+y^{2}-1849\right)(2 x-94) \\
& =0
\end{aligned}
$$

\begin{tabular}{|c|c|c|c|}
\hline No. Anchors & Linear & C-M & Geometric \\
\hline 3 & {$\left[\begin{array}{c}-4.8493 \\
5.1347\end{array}\right]$} & {$\left[\begin{array}{l}0.8825 \\
1.0590\end{array}\right]$} & {$\left[\begin{array}{c}0.8825 \\
1.0590\end{array}\right]$} \\
\hline 4 & {$\left[\begin{array}{c}1.8971 \\
-0.0505\end{array}\right]$} & N/A & {$\left[\begin{array}{c}-0.0150 \\
1.2858\end{array}\right]$} \\
\hline 5 & {$\left[\begin{array}{c}-0.6306 \\
-0.1361\end{array}\right]$} & N/A & {$\left[\begin{array}{c}0.2211 \\
0.0710\end{array}\right]$} \\
\hline
\end{tabular}

THE ESTIMATED POSITION OF NODE 0. THE METHOD FROM [3] IS LABELED "LINEAR", THE METHOD FROM [4] IS LABELED "C-M", AND THE METHOD INTRODUCED IN THIS PAPER IS LABELED "GEOMETRIC".

$$
\begin{aligned}
\frac{\partial J}{\partial y} & =4\left(x^{2}+y^{2}-1225\right) y \\
& +2\left((x-43)^{2}+(y-7)^{2}-1764\right)(2 y-14) \\
& +4\left((x-47)^{2}+y^{2}-1849\right) y \\
& =0
\end{aligned}
$$

The real solutions to this system of equations construct the set, $\mathcal{V}_{\mathbb{R}}=\left\{[3.43,0.85]^{\top},[13.90,31.79]^{\top},[18.22,-29.24]^{\top}\right\}$, and by inspection $p$ is found to be $[18.22,-29.24]^{\top}$. Comparing with the actual position of sensor node $0, p^{*}=$ $[17.9719,-29.3227]^{\top}$ it is seen that the estimate is considerably accurate. The result obtained here is the same as the one calculated in [4]. However here the steps for calculating the solution are less than those used in [4]. Furthermore, smaller number of floating point operations are used here which increases the robustness of the method to numerical perturbations.

Example 6.2: In this example we consider the 3dimensional case. Consider 4 anchors at positions, $p_{1}=$ $[10,13,14]^{\top}, \quad p_{2}=[20,5,40]^{\top}, \quad p_{3}=[12,15,-10]^{\top}$, and $p_{4}=[0,32,5]^{\top}$. Furthermore, sensor node 0 senses its distance to these anchors to be $d_{1}=22.4388$, $d_{2}=46.0740, d_{3}=22.8327$, and $d_{4}=33.3214$. Here, $\mathcal{V}=\left\{[19.73,4.92,-5.27]^{\top},[29.95,20.31,-1.32]^{\top}\right.$ ,$\left.[-1.29,-0.79,-0.38]^{\top}\right\}$, and $p=[-1.29,-0.79,-0.38]^{\top}$ is the global minimum of $J$. Our estimate is very close to the actual position of $0,[0,0,0]^{\top}$.

Example 6.3: In this example we compare the methods from [3], [4], and the one introduced here. Consider 5 anchors, $1, \ldots, 5$, are positioned at, $p_{1}=[10,31]^{\top}, p_{2}=[12,45]^{\top}$, $p_{3}=[-9,10]^{\top}, p_{4}=[30,-3]^{\top}$, and $p_{5}=[-7,53]^{\top}$. The measured distances by the sensor node 0 to each of the anchors corrupted with a zero mean Gaussian noise with variance of $1 \mathrm{~m}^{2}$ are; $d_{1}=32.1404, d_{2}=44.9069, d_{3}=13.5789$, $d_{4}=30.4373$, and $d_{5}=52.3138$. The actual position of node 0 is $p^{*}=\left[\begin{array}{ll}0, & 0\end{array}\right]^{\top}$. The estimated values for each of the methods using 3, 4, and 5 distance measurements are accessible from Tables I and II.

Example 6.4: In this example we compare the result obtained by applying our approach to the example introduced in [2]. Consider $n=5$ anchors in $\mathbb{R}^{2}$ at $p_{1}=\left[\begin{array}{ll}6,4\end{array}\right]^{\top}$, 


\begin{tabular}{|c|c|c|c|}
\hline No. Anchors & Linear & C-M & Geometric \\
\hline 3 & 7.0626 & 1.3785 & 1.3785 \\
4 & 1.8977 & N/A & 1.2859 \\
5 & 0.6452 & N/A & 0.2323 \\
\hline \multicolumn{4}{|c}{ TABLE II }
\end{tabular}

THE ERROR BETWEEN THE ESTIMATED POSITION OF NODE 0 AND ITS ACTUAL POSITION.

$p_{1}=\left[\begin{array}{ll}0, & -10\end{array}\right]^{\top}, p_{1}=\left[\begin{array}{ll}5, & -3\end{array}\right]^{\top}, p_{1}=\left[\begin{array}{ll}1, & -4\end{array}\right]^{\top}$, and $p_{1}=[3,-3]^{\top}$. The distance measurements corrupted by a zero mean Gaussian noise with variance equal to 0.1 are $d_{1}=8.0051, d_{2}=13.0112, d_{3}=0.1138, d_{4}=7.7924$, and $d_{5}=8.0210$. The result obtained from applying RLS and SR-LS from [2] for $p$ are $[-1.9907,3.0474]^{\top}$ and $[-2.018,2.9585]^{\top}$. Applying the method from this study we obtain the same value as SR-LS.

Example 6.5: In this example we consider another example presented in [2]. Consider 5 anchors and an extra anchor at the origin. The range difference measurements corrupted by a zero mean Gaussian noise with the variance of 0.2 are, $\delta_{1}=11.8829, \delta_{2}=0.1803, \delta_{3}=4.6399, \delta_{4}=11.2402$, and $\delta_{5}=10.8183$. The result for $p$ solving (14) is $p=$ $[-4.9798,10.2786]^{\top}$, which agrees with the one obtained in [2].

In the next example, we demonstrate the use of SOS methods in localization. Before stating the numerical example, we first define the general problem of interest which is the modified version of the problem introduced in [13], [14].

Problem 6.1: Consider two mobile sensory agents 1 and 2. Agent 1 moves through a sequence of $n \geq 4$ known different positions, $p_{1}, p_{2}, \cdots, p_{n}$ and agent 2 moves through a sequence of $n$ unknown positions, $q_{1}^{*}, q_{2}^{*}, \cdots, q_{n}^{*}$, where $n \in \mathbb{Z}^{+}$. Their inter-agent distance, $d_{i}$ is measured at each of these positions, where $i \in\{1,2, \cdots, n\}$. In addition agent 2 is capable of estimating its displacement with respect to its initial position using dead-reckoning (odometry), i.e. $\left\|q_{i}-q_{1}\right\|$, $i \in\{1, \cdots, n\}$ are known. We denote $\left\|q_{i}-q_{1}\right\|$ by $r_{i}$. The task is to find estimates of $q_{i}^{*}, \bar{q}_{i}, i \in\{1, \cdots, n\}$ using this information.

The solution to Problem 6.1, $\bar{q}=\left[\bar{q}_{1}^{\top}, \bar{q}_{2}^{\top}, \cdots, \bar{q}_{n}^{\top}\right]$, is the minimizer of the following polynomial function.

$$
\begin{aligned}
J^{\prime}(q) & =\sum_{i=1}^{n}\left(\left\|p^{i}-q_{i}\right\|^{2}-d_{i}^{2}\right)^{2} \\
& +\sum_{i, j \in\{1, \cdots, n\}}\left(\left\|q_{1}-q_{i}\right\|^{2}-r_{i}^{2}\right)^{2} .
\end{aligned}
$$

In other words,

$$
\bar{q}=\operatorname{argmin} J^{\prime}(q) .
$$

Solving (23) using partial derivatives and equating them to zero requires solving a system of polynomial equations with $2 n$ unknowns and $2 n$ equations and even for $n=4$ usual quasi newton methods are not capable of solving it. However using SOS relaxation we can solve it efficiently. This is shown in an example in the next section.

Example 6.6: In this example we consider $p_{1}=\left[\begin{array}{ll}0,0 & 0\end{array}\right]^{\top}$, $p_{2}=[2,-1]^{\top}, p_{3}=[3,2]^{\top}, p_{4}=[4,1]^{\top}, d_{1}=7.0996$, $d_{2}=5.8686, d_{3}=6.8495, d_{4}=4.9226, r_{2}=2.4711$, $r_{3}=5.0914$, and $r_{4}=7.7472$. The distance measurements are noisy, and the noise is considered to be a random Gaussian variable with zero mean and variance equal to 0.1 $\mathrm{m}^{2}$. Solving the optimization problem corresponding to it we obtain, $q_{1}=\left[\begin{array}{ll}0.8732, & 7.0456\end{array}\right]^{\top}, q_{2}=\left[\begin{array}{ll}2.0427, & 4.8685\end{array}\right]^{\top}$ $q_{3}=\left[\begin{array}{ll}5.8222, & 8.2410\end{array}\right]^{\top}$, and $q_{4}=\left[\begin{array}{ll}7.9623, & 3.9208\end{array}\right]^{\top}$, Comparing with the real values; $[1,7]^{\top},[2,5],[6,8]$, and $[8,4]$, respectively, we observe that estimates are very close to the real values.

\section{Comparison with the Existing Methods}

The formulated localization problem as in [4] with $n$ anchors can be described as what comes next. Let $\varepsilon_{i}$ be the error in the estimated squared distances between sensor 0 and anchor $i$. We want to minimize the sum of squared errors

$$
J_{C M}=\varepsilon_{1}^{2}+\varepsilon_{2}^{2}+\ldots+\varepsilon_{n}^{2},
$$

subject to $n-2$ equality constraints

$$
f_{i}\left(\varepsilon_{1}, \varepsilon_{2}, \varepsilon_{i}\right)=0, \quad i=3,4, \ldots, n .
$$

where $f_{i}$ are obtained from writing different Cayley-Menger determinants for different set of anchor nodes. Using Lagrange multiplier method this minimization is equivalent to minimizing

$H\left(\varepsilon_{1}, \ldots, \varepsilon_{n}, \lambda_{1}, \ldots, \lambda_{n-2}\right)=\sum_{i=1}^{n} \varepsilon_{i}^{2}+\sum_{i=1}^{n-2} \lambda_{i} f_{i+2}\left(\varepsilon_{1}, \varepsilon_{2}, \varepsilon_{i+2}\right)$.

One way of solving the above minimization problem is to differentiate equation (26) with respect to variables $\varepsilon_{i}$ and $\lambda_{i}$, and to find the zeros of these differentials. In this process we have $2 n-2$ equations, each with degree 2 , that need to be solved using a nonlinear root finding method. Hence, since in our proposed method adding anchors have no effect on the number of equations and variables, we always have a system of polynomial equations comprising of 2 equations and 2 unknowns.

For three anchors the results obtained using the method introduced here and the one in [4] are the same and are clearly better than the result obtained from linear algorithm of [3].

The result obtained by applying SR-LS method from [2] and the method introduced here give the same numerical result. However, in solving SR-LS positive definiteness of a certain matrix is assumed while here no assumption is in place.

For more than 3 anchors the method introduced in [4] does not result in a solution using the fsolve routine in MATLAB, which uses a quasi-Newton algorithm to solve a set of nonlinear equations. The method introduced in this paper, however, yields a result that is more accurate comparing with the one obtained by the linear method in [3].

In the case of localization using range difference measurements the biggest difference between the method introduced here and the one in [2] is the number of steps to reach the final result. While, in this paper we only need to solve one minimization problem, there to find the result one needs to go through a 4 stage algorithm. 


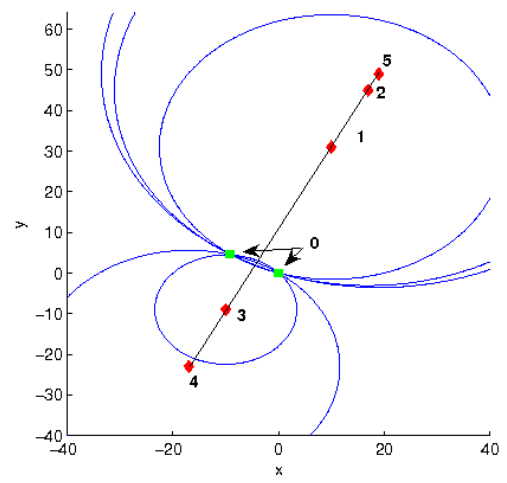

Fig. 3. The localization problem with colinear anchors.

\begin{tabular}{|c|c|c|c|}
\hline No. Anchors & 3 & 4 & 5 \\
\hline$\left[\begin{array}{l}\mathrm{x} \\
\mathrm{y}\end{array}\right]$ & {$\left[\begin{array}{c}-3.9977 \\
3.0045\end{array}\right]$} & {$\left[\begin{array}{c}-3.9916 \\
3.0169\end{array}\right]$} & {$\left[\begin{array}{c}-3.9911 \\
3.0177\end{array}\right]$} \\
\hline
\end{tabular}

TABLE III

THE ERROR BETWEEN THE ESTIMATED POSITION OF NODE 0 AND ITS ACTUAL POSITION.

\section{COLINEAR ANCHORS}

In this section we discuss the situation (in $\mathbb{R}^{2}$ ) where the anchors are colinear. Consider $n$ colinear anchors labeled 1,2 to $n>N$ at positions $p_{i} \in \mathbb{R}^{2}$, where $i \in\{1, \cdots, n\}$. Furthermore, sensor node 0 can measure its distance $d_{i}$ from the anchor $i$. The geometric representation of a problem with 5 colinear anchors in $\mathbb{R}^{2}$ is depicted in Fig. 3. In the case where the distances are exact there are two points of intersection for the circles, however in the presence of noise the circles will not necessarily intersect at any common intersection point. Defining $J(p)$ as before and solving (8), we can compute an estimate for the position of node 0 , and since the anchors are colinear the other possible position for node 0 is the mirror of this point where the line connecting the anchors is the mirror axis.

Using the method here one can have estimates for the two possible positions of node 0 while the other methods in the literature fails completely when the anchors are colinear.

Example 8.1: Consider the problem of localization as depicted in Fig. 3. The anchors are placed at $p_{1}=[10,31]^{\top}$, $p_{2}=[17,45]^{\top}, p_{3}=\left[\begin{array}{ll}-17, & -23\end{array}\right]^{\top}, p_{4}=\left[\begin{array}{ll}-10, & -9\end{array}\right]^{\top}$, and $p_{5}=[19,49]^{\top}$. Again, it is considered that distance measurements are being taken by sensor node 0 in the presence of a zero mean Gaussian noise with variance of $1 \mathrm{~m}^{2}$. The actual position of sensor node 0 is at $[0,0]^{\top}$. The estimates of the node 0 position (the ones closer to the actual position) using 3,4, and 5 distance measurements are presented in Table III.

\section{Concluding Remarks and Future Directions}

A polynomial method is introduced for addressing network localization problems with noisy inter-sensor measurements.
The method is applied to different scenarios and the results obtained are compared with the results obtained by applying other methods in the literature. As demonstrated later in the paper, our method applies to non-generic scenarios with colinear anchors as well.

The immediate potential future research is the extension of the introduced methodologies to scenarios where more than one node is needed to be localized.

\section{ACKNOWLEDGMENT}

This work is supported by NICTA, which is funded by the Australian Government as represented by the Department of Broadband, Communications and the Digital Economy and the Australian Research Council through the ICT Centre of Excellence program.

\section{REFERENCES}

[1] J. V. Carroll, "Vulnerability assessment of the u.s. transportation infrastructure that relies on the global positioning system," The Journal of Navigation, vol. 56, pp. 185-193, 2003.

[2] A. Beck, P. Stoica, and J. Li, "Exact and approximate solutions of source localization problems," IEEE Transactions on Signal Processing, vol. 56, pp. $1770-1778,2008$.

[3] A. Sayed and A. Tarighat, "Network-based wireless location," IEEE Signal Processing Magazine, p. 2440, July 2005.

[4] M. Cao, B. D. O. Anderson, and A. S. Morse, "Sensor network localization with imprecise distances," Systems and Control Letters, vol. 55, pp. 887-893, November 2006.

[5] F. Thomas and L. Ros, "Revisiting trilateration for robot localization," IEEE Transactions on Robotics, vol. 21, no. 1, pp. 93-101, Feb 2005.

[6] K. Whitehouse, C. Karlof, A. Woo, F. Jiang, and D. Culler, "The effects of ranging noise on multihop localization: an empirical study," Information Processing in Sensor Networks, 2005. IPSN 2005. Fourth International Symposium on, pp. 73-80, April 2005.

[7] I. Shames, S. Dasgupta, B. Fidan, and B. D. O. Anderson, "Circumnavigation using distance measurements," 2009, to appear in Proc. of European Control Conference.

[8] G. Mao, B. Fidan, and B. Anderson, "Wireless sensor network localization techniques," Computer Networks, vol. 51, pp. 2529-2553, 2007.

[9] C. B. Garcia and T. Y. Li, "On the number of solutions to polynomial systems of equations," SIAM Journal on Numerical Analysis, vol. 17, no. 4 , pp. $540-546$.

[10] P. A. Parrilo and B. Sturmfels, "Minimizing polynomial functions," DIMACS Series in Discrete Mathematics and Theoretical Computer Science, vol. 60, pp. 83-99, 2003.

[11] P. Parrilo, "Semidefinite programming relaxations for semi-algebraic problems," Mathematical Programming, vol. 96, no. 2, pp. 293-320.

[12] D. Henrion and J. Lasserre, "Detecting global optimality and extracting solutions in gloptipoly," in In Positive Polynomials in Control, ser Lecture Notes on Control and Information Sciences, D. Henrion and A. Garulli, Eds. Springer-Verlag, 2005

[13] I. Shames, B. Fidan, B. D. O. Anderson, and H. Hmam, "Cooperative self-localization of mobile agents moving in planar formations," provisionally accpeted to appear in Transactions on Robotics.

[14] X. S. Zhou and S. I. Roumeliotis, "Robot-to-robot relative pose estimation from range measurements," IEEE Transactions on Robotics. vol. 24, no. 6, pp. 1379-1393, 2008. 